\title{
Variable allocation of activity to daylight and night in the mallard
}

\author{
Pius Korner ${ }^{\mathrm{a},}{ }^{*}$, Annette Sauter ${ }^{\mathrm{a}}$, Wolfgang Fiedler ${ }^{\mathrm{b}}$, Lukas Jenni ${ }^{\text {a }}$ \\ ${ }^{a}$ Swiss Ornithological Institute, Sempach, Switzerland \\ ${ }^{\mathrm{b}}$ Max Planck Institute for Ornithology, Radolfzell, Germany
}

Keywords:

activity budget

Anas platyrhynchos

diel rhythm

flexible activity allocation

mallard

moon

weather
The solar day-night rhythm imposes a strict diel activity pattern on many organisms. Among birds, most species are generally either active during the day and rest during the night, or vice versa. However, many waterbird species can be active during both daylight and darkness. Hence, these species are much less limited by an external clock to allocate their activities over time than species showing a strict day or night pattern. Before miniaturized data-logging systems became available, it was difficult to follow animals day and night. Therefore, few details about short-term activity budgets are available for free-living animals. To study the activity budget of mallards, Anas platyrhynchos, in relation to time of the day, season and external factors, we used tags containing an accelerometer providing detailed activity information. We observed a relatively constant diel pattern with more activity during daylight than at night and peak activities during twilight. Activity over the season (September-April) was remarkably constant. Compared to the average activity per half-day, excess activity alternated every $12 \mathrm{~h}$, suggesting an increased need for rest during daylight after a night with excess activity, and vice versa. Between days, activity was allocated to half-days in a very flexible manner: Either day or night activity was increased for a number of days, before increased activity gradually switched to the other half-day. We observed no synchrony in this pattern among individuals, highlighting the behavioural flexibility of this generalist species.
When to be active versus inactive may be seen as one of the most fundamental mechanisms to modulate life functions in most or all organisms (Dunlap, Loros, \& DeCoursey, 2004). The decision when to be active is often strongly influenced by the rhythms induced by the earth's and the moon's movements. There are diel and annual, lunar and tidal rhythms, the two former being the most influential (Daan \& Aschoff, 1975; Gwinner, 1975). Activity patterns governed by these rhythms are fine-tuned, responding to more immediate influences that may or may not be rhythmic, e.g. physiological state, or external factors such as weather conditions, presence of food, or presence and behaviour of enemies or conspecifics (Kronfeld-Schor \& Dayan, 2003; Sharma \& Chandrashekaran, 2005).

Most bird species can be categorized as diurnal or nocturnal according to their diel rhythm, each category using one of the two half-days for their main activity (for the work presented here, we define 'active' as nonminor body movements). The majority of species are diurnal, with the notable exception of nocturnal

\footnotetext{
* Correspondence: P. Korner, Swiss Ornithological Institute, Seerose 1, 6204 Sempach, Switzerland.

E-mail address: pius.korner@bluewin.ch (P. Korner).
}

migration or nocturnal singing. Some species are nocturnal, e.g. owls and nightjars (Martin, 1990). Finally, a minority of bird species have evolved to regularly use daylight as well as the darkness for their main activities, primarily including waders and waterbirds. One reason for a variable day-night activity pattern is the tidal rhythm, exposing profitable foraging sites during low tide, which is not linked to the diel rhythm. But nocturnal as well as diurnal activity is observed in many duck species away from marine shorelines, too (e.g. Martin, 1990; Merke \& Mosbech, 2008; Owen, 1991). In these species, one of the major activities observed during the night is feeding. Night feeding has been linked to the avoidance of predators, hunting or other types of disturbance, as well as to increased food availability (e.g. insects active or emerging at night; McNeil, Drapeau, \& Goss-Custard, 1992; Ydenberg, Prins, \& Van Dijk, 1984), or to the need for additional foraging time when the daylight period is too short to fulfil food requirements (McNeil et al., 1992). Guillemain, Fritz, and Duncan (2002) reversed the argument and noted that daytime feeding was sometimes needed to supplement the otherwise preferred night feeding. Independent of when ducks are feeding, peak activities are usually observed during twilight, e.g. for commuting flights (Johnson, Schmidt, \& Tylor, 2014; Tamisier, 1978). 
Seasonal patterns are as apparent as are diel patterns. Reproduction, moult and migration are some of the activities linked to specific seasons in birds. Also, daily behavioural patterns such as the time devoted to foraging may change seasonally: Arzel, Elmberg, and Guillemain (2007) showed that diurnal foraging increased dramatically during moult and migration stopovers. Lunar rhythms schedule life in the intertidal zone. Other lunar rhythms, however, are less well known in bird species, and are primarily described as increased activity on moonlit nights around full moon (e.g. Mayhew, 1988; McNeil et al., 1992; Zavalaga, Dell'Omo, Becciu, \& Yoda, 2011).

Apart from these rhythmic external factors, more or less unpredictable factors influence the activity level of animals, e.g. weather parameters. In waterbirds, colder weather may first increase (feeding) activity, but activity may be decreased at temperatures below freezing to save energy (Paulus, 1988; Quinlan \& Baldassarre, 1984). Strong winds or rain may also reduce activity when ducks seek sheltered places (Paulus, 1988).

Before miniaturized accelerometers became available (see e.g. Shepard et al., 2008), it was often not possible to follow free-living individuals over prolonged periods. Highly mobile or nocturnal species were especially difficult to study. Hence, the $24 \mathrm{~h}$ pattern of activity and its variability between and within individuals are not well documented for such species. Here we present activity data from wintering mallards, Anas platyrhynchos. We focus on the allocation of activity to daylight hours versus night hours, and on the influence of weather conditions and the moon on activity.

The mallard is the most common and widespread dabbling duck species in Europe (Bauer, Bezzel, \& Fiedler, 2005). It is well adapted to anthropogenic landscapes and it uses human settlements for breeding and wintering (e.g. Kozulin, 1995; Pulliainen, 1963). Agricultural fields can be an important food source, e.g. for grains or potatoes (Thomas, 1982). In central Europe, individuals from further north and northeast join the local breeders in winter (Scott \& Rose, 1996). During cold spells, ducks may escape freezing conditions on lake shores by moving to rivers (A. Sauter, personal observation) or by migrating southwestwards (Hofer, Korner, \& Korner-Nievergelt, 2010; Sauter, Korner-Nievergelt, \& Jenni, 2010).

In Sauter, Korner, Fiedler, and Jenni (2012) we presented diel short-distance movements and general diel activity patterns of mallards, using individuals tagged with traditional VHF tags as well as individuals with automated positioning and movement registrations ('GPS tags' with accelerometers for activity measurements). Here we present more detailed analyses of the same activity data (seven individuals with GPS tags), together with new data from individuals from southern Germany (eight individuals) equipped with similar tags.

\section{METHODS}

\section{Study Sites}

Ducks were caught in Oberkirch $\left(47^{\circ} 9^{\prime} 47^{\prime \prime} \mathrm{N}, 8^{\circ} 7^{\prime} 21^{\prime \prime} \mathrm{E}\right)$ and Wauwiler Moos $\left(47^{\circ} 10^{\prime} 23^{\prime \prime} \mathrm{N}, 8^{\circ} 1^{\prime} 5^{\prime \prime} \mathrm{E}\right)$, both in central Switzerland (500 $\mathrm{m}$ above sea level, $8 \mathrm{~km}$ apart), and in Radolfzell, southern Germany $\left(47^{\circ} 45^{\prime} 58^{\prime \prime} \mathrm{N}, 8^{\circ} 59^{\prime} 51^{\prime \prime} \mathrm{E}, 400 \mathrm{~m}\right.$ above sea level). The Swiss ducks used Lake Sempach and surroundings, the German ducks mainly used Mindelsee and Untersee (the western part of Lake Constance); henceforth, we name our two study sites 'Lake Sempach' and 'Lake Constance', respectively. All ducks used an area expanding up to about $10 \mathrm{~km}$ across, consisting of a mixture of agricultural land (mainly grassland, some crop and maize) interspersed with woods and small to medium-sized settlement areas (up to 20000 inhabitants). At Lake Constance reed beds were used to a minor degree. Both sites were mostly flat, bordered by hills rising $200-300 \mathrm{~m}$ above the lakes. Both sites provided various water bodies (lakes, ponds, rivers, canals) and feeding opportunities on shores and agricultural fields. Limited public feeding was conducted on some lake shores, but we do not know whether and how much our ducks used this food source.

At both sites, duck hunting was practised at low levels only; possibly, the ducks we observed avoided certain areas at certain times due to hunting. None the less, we have no indication for hunting strongly influencing the behaviour of the individuals studied.

\section{Catching and Telemetry}

Individuals were caught in baited traps at the Lake Sempach site (see Sauter et al., 2012 for details) in 2009 and with a spoon net at the Lake Constance site in 2012 (see Fig. 1). After sexing, weighing and ringing, the ducks were equipped with a GPS data logger containing an accelerometer and VHF transmitter (E-Obs Digital Telemetry, Gruenwald, Germany) with a harness of 25-inch tubular Teflon (Bally Ribbon Mills, Bally, PA, U.S.A.); the total mass was $44 \mathrm{~g}$ (all ducks weighed at least $1000 \mathrm{~g}$ ). The harness was designed as described in Roshier, Klomp, and Asmus (2006). A $3 \mathrm{~cm}$ long piece of shrinkable tubing with a diameter of $3 \mathrm{~mm}$ (Klebeschrumpfschlauch; product no. 121-0512, Farnell, Zurich, Switzerland) was used to form the T-loop on the breast.

The tags recorded the acceleration in three dimensions during bouts of $4-17 \mathrm{~s}$ (depending on the settings of the tag), registering $82-180$ acceleration measures in each of the three directions during each measurement bout. Measurement intervals were $1-5 \mathrm{~min}$, depending on the tag. The different settings of the tags are unlikely to have influenced the results, because, as described below, we used the variance of the acceleration measurements per measurement bout for the analyses. In addition, we aggregated these values for periods of about $1 \mathrm{~h}$. Hence, data from tags with longer measurement bouts and/or more measurements per time unit are somewhat more precise, but there is no bias in our measure depending on the tag settings. The data stored on the tags were downloaded remotely at least weekly using the Base station II (Eobs). A Yagi-antenna and radio receiver (Yaesu VR-500; Vertex Standard, Cyprus, CA, U.S.A.) were used to localize the duck for data downloading; the tags emitted a ping signal during specific periods of the day.

At Lake Sempach, nine ducks were equipped with a tag, but two were not analysed, one because acceleration was not measured during the night and one because it was the partner of another duck in the sample. Ducks were followed until contact with the tag was lost, or until the end of March 2009. At Lake Constance, we included data from eight ducks that appeared not to be semidomesticated (e.g. one excluded duck only used one pond and depended on human feeding) until 29 April 2013 (Fig. 1). Thus, all ducks analysed here were not tame, albeit they were accustomed to living in an anthropogenic landscape.

\section{Sun, Moon and Weather Data}

Times for sunrise, sunset and twilight periods (civil, nautical and astronomical twilight) were taken from http://cmslive3.unibe.ch/ unibe/philnat/aiub/content/services/index_eng.html for Zurich. To account for the different longitudes of the study sites, 1.5 min was added for the Lake Sempach data and 1.5 min was subtracted for the Lake Constance data. Data on moonrise, moonset, full and new moon were taken from the same web source. We assigned a moon sickle value of 0 for new moon, 1 for full moon, and a sinusoid interpolation for waxing and waning in between (value calculated per night). Weather data originated from the weather stations in 

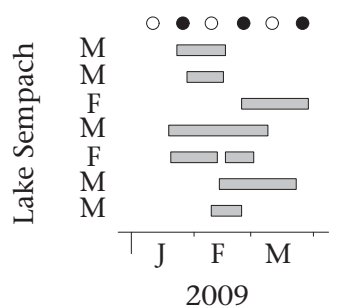
- New moon
- Full moon
$\square$ Periods with data (for each individual)
F Female
M Male

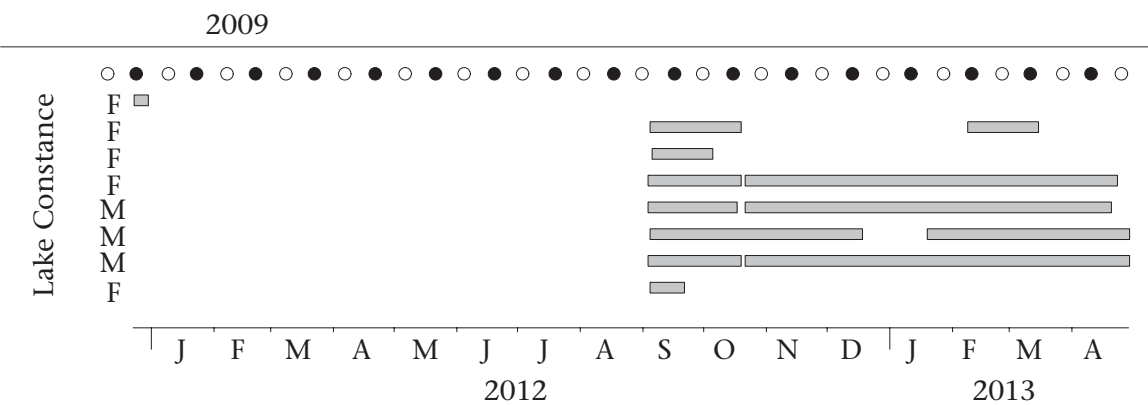

Figure 1. Duration of data collection per individual (horizontal grey bars, some individuals with gaps). For each of the two sites, the dates of new moon and full moon as well as the sex of the individual are indicated.

Lucerne (10 km southeast of Lake Sempach) and Schaffhausen (25 km west of the Lake Constance site). We used total rain per h, mean temperature per $\mathrm{h}$ and mean wind speed per $\mathrm{h}$ and assigned the corresponding values to each acceleration measurement bout (i.e. all bouts per $\mathrm{h}$ and from the same site had the same weather values). In the model, rain and wind values were log-transformed and temperature was standardized ( $z$-transformation).

\section{Data Processing and Analyses}

Data processing and analyses were conducted with R3.2.0 for Windows (R Core Team, 2015). For each measurement bout (of 4-17 s), we calculated the variance of the acceleration measurements of each axis and used the mean of these three variance values as a measure of activity of the duck (called 'activity measure'). The distribution of these activity measures was strongly bimodal, indicating resting versus (any type of) activity. Owing to strong autocorrelation, we did not analyse single bouts; rather, we aggregated the activity measures. To that end, we used the arithmetic mean of the activity measures per 10th of the daylight period (sunrise to sunset), per 10th of the night (excluding twilight) and per twilight period (three each for dawn and dusk), resulting in 26 'day periods' each lasting about $1 \mathrm{~h}$ (for daylight and night) or about $30 \mathrm{~min}$ (for the twilight periods). Weather parameters were aggregated in the same way. For some analyses, values were further aggregated per daylight and night (excluding the twilight periods, hence yielding two values per $24 \mathrm{~h}$ day).

Among the bouts with activity, we could readily discern bouts of flight by their clear alternating accelerations, especially on the $\mathrm{Z}$ axis (heave; Fig. A1a). We first identified candidate flight bouts by their very pointed energy peak above $6 \mathrm{~Hz}$ in the energy spectral density (which was derived using a fast Fourier transform). Then, the acceleration profiles of these preselected bouts were visually examined to confirm flight. Other specific activities could not be reliably differentiated due to the large variability in the acceleration patterns (Fig. A1b-e).

To estimate the mean activity per day period, we fitted a linear mixed model with day period (levels $1-26$ ) as the only fixed factor (the outcome variable was, as described above, the mean of the activity measures per single day period). The identity of the individual and the day of the year (1-365) were used as two crossed random factors, and a lag-one autoregressive correlation structure was assumed for the residuals. The model was fitted with the function glmmPQL, using list(const $=$ pdBlocked(list(pdIdent( ind-
1), pdIdent( dayofyear-1)))) to specify the crossed random factors as suggested by D. Bates (stat.ethz.ch/pipermail/r-help/2003-April/ 032673.html; const is a factor with all ones and length equal to the number of data points, ind and dayofyear are the factor names). A binomial model with the number of bouts with a flight versus the number of bouts without a flight per day period as the dependent variable and with the same fixed, random and autocorrelation structure as above was fitted to estimate the average probability of flight per day period. Mean daily activity over the season was estimated using a similar model as the model for activity per day period, but with the day of the year as the fixed term, and the individual and day period as crossed random terms (and, again, a lagone autoregressive correlation structure).

We built analogous glmmPQL models for the weather and moon parameters. First, we built one model for each site using the night data only. Fixed factors were temperature, rain, wind, moon sickle (between 0 and 1) and moon position (below or above horizon; day periods with a moon rise or moon set were excluded). The starting models included the quadratic terms of moon sickle and temperature (owing to a possible nonlinear temperature effect as outlined above), as well as the interaction term between moon sickle and moon position. Quadratic terms and the interaction were excluded if a term was not supported by the approximate $t$ tests of the parameters (summary output of the glmmPQL object). Further model selection was not conducted, but we drew effect plots to judge the importance of a predictor variable. To achieve this, the prediction was plotted for average values of these model parameters not shown in the effect plot, and an uncertainty estimate was calculated using the delta method (function deltaMethod in R). In the final model, there was still some autocorrelation, especially around lag 10, i.e. residuals showed some correlation with the residual from the same day period of the previous night (partial autocorrelation was around 0.05 ). To explore the effect of this autocorrelation, we fitted a model using only the odd days of the year (results not shown), which reduced the autocorrelation pattern. All effects that had been strongly supported according to the $P$ value in the model with all dates were still present in this analysis based on the thinned data set. Finally, to explore whether the effects of the weather parameters observed in the night data also held for the rest of the day, we built a similar weather model for each site using the daylight and twilight data, but excluding the moon parameters.

To compare the activity per half-day (daylight versus night, excluding twilight), we calculated the expected mean activity per 
half-day, corrected for season (day of year up to its third polynomial), rain, temperature and wind speed (mean weather values for the measurements of the corresponding half-day; normal linear model, separately for each duck individual, with a lag-oneautocorrelation modelled in the residuals; function gls in R). We then plotted the residuals of the observed value to this fitted value against time.

\section{Ethical Note}

Tagging was performed by trained personnel and animals released as quickly as possible. Individuals weighing less than $1000 \mathrm{~g}$ were not used for the study. From direct field observations and from our data, we have no indication of the tags or harnesses notably disturbing the individuals. Individuals were not recaught for tag removal; some individuals retained the tags and data continued to be collected. A few tags were lost and found, while the fate of the other tags remained unknown. Catching and telemetry permissions were granted by Landwirtschaft und Wald Canton of Lucerne, Swiss Federal Office for the Environment, and for the German state of Baden-Württemberg by Regierungspräsidium Freiburg, departments of agriculture (for animal experiment permits) and environment (for trapping permit).

\section{RESULTS}

\section{Diel and Seasonal Activity Patterns}

Independent of site and season, the daily activity pattern showed two distinct peaks, one very pronounced peak during the evening nautical twilight, and one peak during nautical and civil twilight in the morning and into the first one or two daylight hours (Fig. 2b). Activity gradually decreased during daylight. During the night, overall activity was reduced, with the lowest activity at the beginning and at the end of the night, before activity sharply increased during nautical twilight. When we included sex as a second predictor in this model, our data suggest that the mean activity value of males was about 0.35 larger than the activity value of females (Fig. A2; compare the effect size with the mean activity in the course of the day, which spans from about $4.5-7$, Fig. 2). Flights were observed mainly during civil and nautical twilight (Fig. 2a). Fewer flights were observed during daylight and very few during darkness. When we recalculated mean activity per day period (as in Fig. 2b) but excluded flights, the pattern remained nearly identical. Hence, the activity peaks during twilight were not merely a result of commuting individuals (e.g. two of the seven ducks from Lake Sempach were regular commuters between two sites 8 km apart, see Korner, Sauter, \& Jenni, 2015).

Activity was remarkably constant across the season (i.e. from September to April). The smoothed activity values (mean daily activity smoothed using a local polynomial regression fit) always lay between about 5.4 and 5.6, which is insignificant compared to the daily variation in the activity (see Fig. 2); daily means were scattered with no apparent pattern, mostly between 5 and 6 .

\section{Weather and Moon}

Among the weather parameters, rain had the clearest effect: activity strongly increased with rainfall in all four models (one model for each site and for night or daylight-twilight; Fig. 3; the corresponding plots for the other three models were qualitatively the same; Table A1). Effects of the other two weather parameters were less conclusive (Table A1). In only one of the four models was a quadratic temperature effect supported: the daytime data from Lake Constance suggested a negatively bent correlation $(P=0.007)$, with less activity at higher temperatures around $20^{\circ} \mathrm{C}$, somewhat increasing activity at cooler temperatures and marginally decreasing activity again when temperatures fell below freezing. In the other three models, no quadratic temperature effect was supported. Daytime data from Lake Sempach showed no effect of temperature on activity, while the night data showed a slight positive correlation for Lake Sempach and a slight negative correlation for Lake Constance. We observed no consistent effect of wind speed: two of the four models showed a slightly positive and two a slightly negative correlation between wind speed and activity.

Effects of the moon on activity, too, were inconclusive and differed between the two sites. In the data from Lake Sempach, an interaction term between moon sickle and moon position on activity was not supported. The approximate $P$ values supported an effect of moon position $(P=0.05)$ and a quadratic effect of moon sickle $(P=0.002)$, suggesting increased activity during times with the moon above the horizon and, independent of moon position, during nights with either a new moon or a full moon (Fig. 4a). The Lake Sempach data covered one to two moon cycles, only (see Fig. 1, Table A1); hence these observations need to be judged with caution. In the data from Lake Constance, extending over a much longer period, the interaction between moon sickle and moon position was retained $(P=0.002)$, but this was not the case for the quadratic term of moon sickle $(P>0.5$ for the quadratic term as main effect and in interaction with moon position). The effect plot suggested that activity increased somewhat during a full moon, but only when the moon was above the horizon (Fig. 4b).

\section{Short- and Medium-term Patterns of Activity per Half-Day}

For the following analyses, we worked with the average activity per half-day, i.e. with the mean value of all activity measures during daylight and during the night (excluding the twilight periods). As shown above, activity during daylight was higher than during the night (Fig. 2). When we corrected for this systematic difference (and, in addition, for season by including the date as a linear, quadratic and cubic term, and the three weather parameters rain, temperature and wind speed), and plotted the residuals against time, there appeared a conspicuous pattern of an alternation of the sign of the residuals between half-days (Figs. 5 and 6): when activity during the day was higher than expected, activity during the following night was often lower than expected; similarly, when activity during the night was higher than expected, activity during the following day was generally lower than expected.

Which half-day (day or night) showed positive residuals (i.e. 'excess' activity) varied within individuals in periods of a few days to weeks: after a number of days of excess night activity there generally followed a period of excess day activity, and vice versa. Some individuals showed some regularity regarding this switching (especially the fourth and fifth individuals depicted in Fig. 5 with a switch about every week), but most individuals showed little regularity. The underlying process seemed to be a gradual increase in the daylight activity correlated with a gradual decrease in the night activity, before daylight activity was reduced again while night activity increased. Hence, our data suggest a gradually varying allocation to daylight or night of a more or less constant total $24 \mathrm{~h}$ activity budget.

Comparing the activity between individuals, we did not find a general synchrony in the residuals (i.e. in the deviation of the activity from the expected value given the half-day, season and weather; Figs. 5 and 6). The mean correlation of the residuals between all pairs of individuals was 0.03 for Lake Sempach (range -0.24 to 0.30 ; only comparisons with at least 10 overlapping halfdays) and 0.11 for Lake Constance ( -0.39 to 0.50 ). We controlled for the three weather parameters in the model; hence, synchronization due to similar responses to these weather parameters would not 

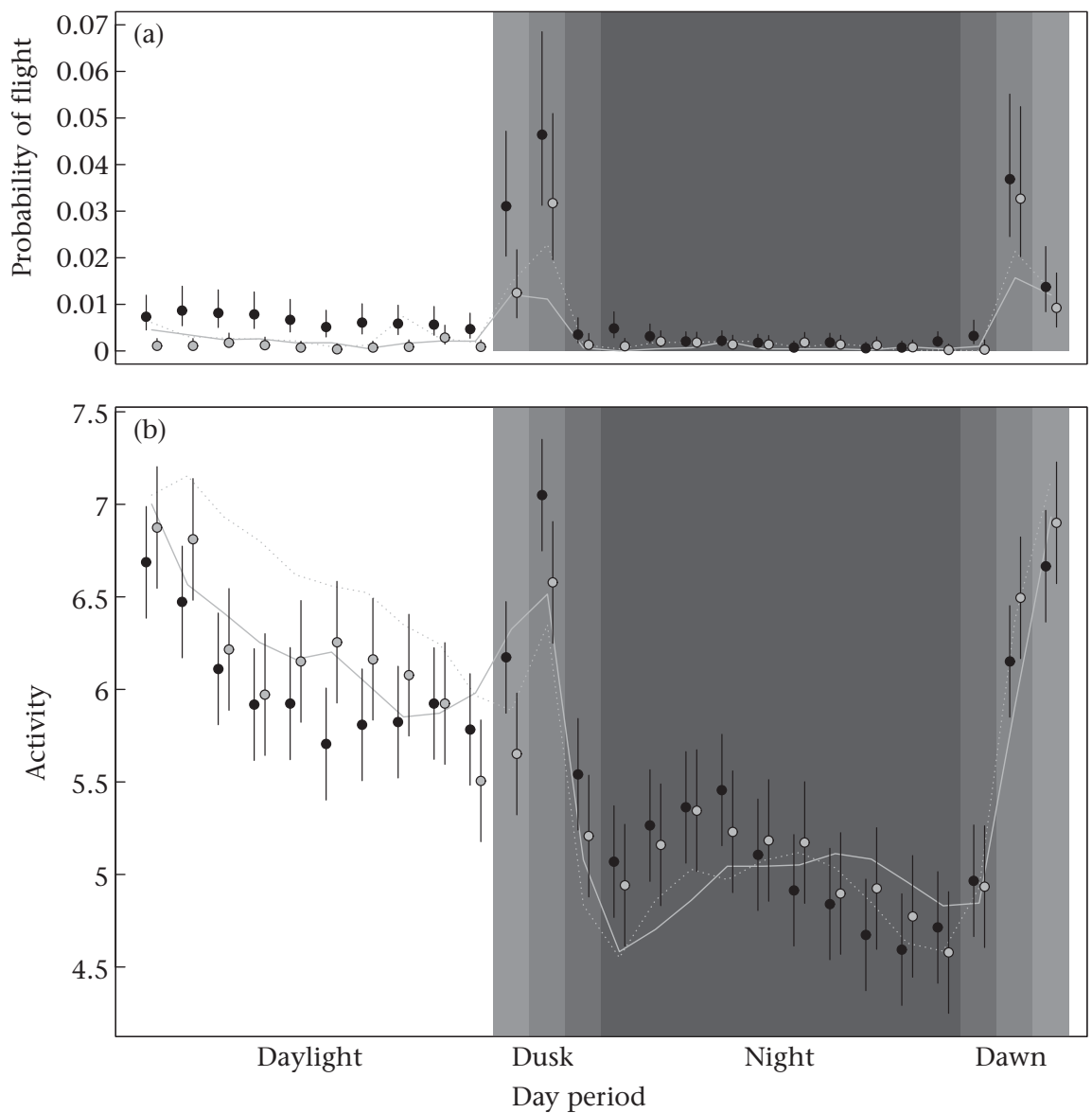

Figure 2. Mean probability of (a) a flight and (b) mean activity over the course of the day. Black dots: ducks from Lake Sempach, 19 January-29 March; grey dots: ducks from Lake Constance, 19 January-29 March; grey lines: ducks from Lake Constance, but different period of the year: 4 September-11 November (solid line) and 12 November-18 January (dotted line). Daylight and night were subdivided into 10 equally long periods. Vertical bars behind dots are \pm 2 SE. SEs for the grey lines were similar but omitted for better readability. For the activity value, we first averaged the variances of the acceleration measurements of the three axes, and, second, averaged these values per individual, date and day period. Histograms of all observed activity values per site are depicted in Fig. 4 and may help to interpret the variation in the activity seen in this graph.
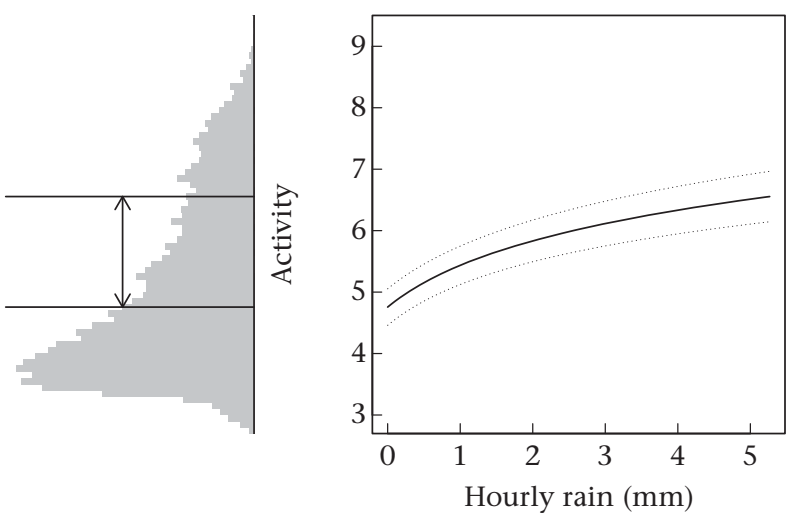

Figure 3. The effect of rain on activity (solid line) with uncertainty intervals of $\pm 2 \mathrm{SE}$ (dotted lines), illustrated with the data from Lake Constance. The other model parameters were set to their averages (moon position to 'below horizon'). The horizontal histogram of the observed activity values (mean of the activity measures per day period; the y-axis of the effect plot also applies to the histogram) helps to interpret the effect size; the double arrow indicates the fitted activity values corresponding to the observed minimum and maximum rain. Effect line and histogram are based on night values only.

have appeared in these correlations. Excluding the weather parameters from the model even decreased the correlation coefficients (0.02 for Lake Sempach and 0.00 for Lake Constance).

\section{DISCUSSION}

This study demonstrates how flexible mallards allocate their activity over the day and night, switching between excess daylight and excess night activity, largely independent of moon, weather and season. This flexibility appears to be the result of modulations of a basic diel pattern which shows clear peaks around twilight and a higher overall activity during daylight than at night. Similarly, Bengtsson et al. (2014) reported a rather strict overall diel activity pattern in the mallard (also shown e.g. by Winner, 1960) that may be modulated to various degrees, while other work showed the general flexibility of this species in different contexts, such as migration behaviour (Diefenbach, Nichols, \& Hines, 1988; van Toor et al., 2013) or movements within the wintering grounds (Sauter et al., 2010; Tamisier, 1978) or during migration stopover (Bengtsson et al., 2014). However, previous studies on mallards, using traditional techniques, have not achieved the resolution of the now available automated recording techniques. On the other hand, studies applying these new techniques have mostly focused on specific behaviours (Shepard et al., 2008) but not on the overall allocation of activity over time.

While the automated recording of behavioural data yields a detailed description of activity patterns, the technique has a number of limitations: only a small number of individuals (15 in our study) can be monitored. It may be difficult to identify precise behavioural types (Shepard et al., 2008; our study), and we lack 
Lake Sempach

(a)

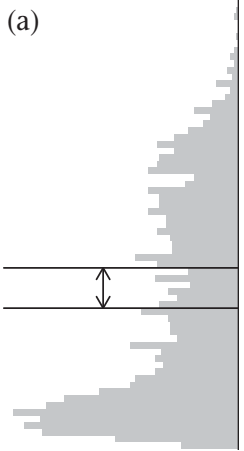

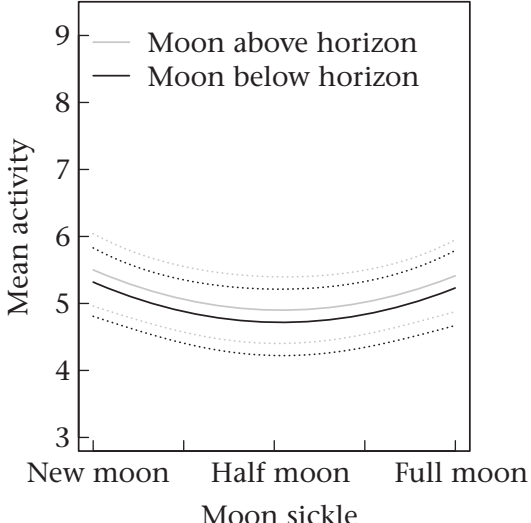

Lake Constance

(b)

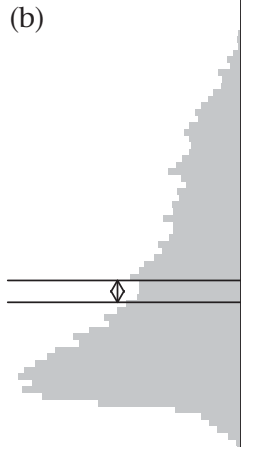

Figure 4. Effect of moon on the activity observed in the data from (a) Lake Sempach and (b) Lake Constance. The interaction between moon sickle and moon position was not supported in the data from Lake Sempach, hence it was excluded from the model. On the other hand, the quadratic term of moon sickle was not supported in the data from Lake Constance. Dotted lines indicate $\pm 2 \mathrm{SE}$. The histograms show the distribution of the activity data from the corresponding lake (the $y$-axis of the effect plot also applies to the histogram); the double arrows indicate the effect size of the moon sickle (shown for 'moon above horizon'; night data only).

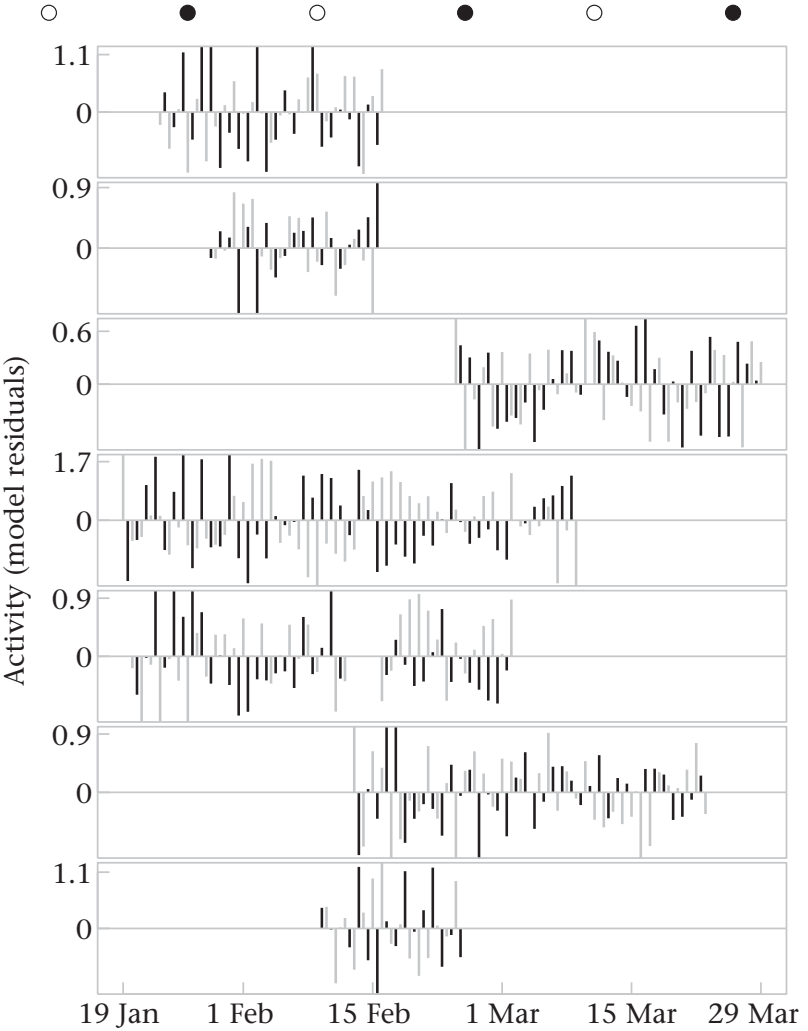

Figure 5. Activity of the ducks from Lake Sempach per half-day (grey=during daylight, black = during the night). Each row corresponds to one duck individual. The values are residuals from a model including half-day (daylight versus night), date (up to the cubic polynomial), rain, temperature and wind speed as predictors and activity (mean per half-day) as the outcome. Hence, the values shown are the deviations from what would be expected given the half-day, the season and the measured weather parameters. For better readability, only two values are given along the y-axes; to judge the effect, values may be compared with activity values shown in Figs. 2-4. Dots above the plot indicate new moon (black) and full moon (white).

knowledge about various covariates such as flock size, disturbance events, etc. that could influence the behaviour.

Despite the fact that the main activity type changes in the course of the year (e.g. migration, moult, courtship, reproduction; Arzel et al., 2007; Raitasuo, 1964), we found little variation in the overall level of activity (recall that 'activity' includes any type of movement of the body) with date. Our data cover the period from September to April, i.e. they do not include incubating or actively migrating individuals when mean activity, of course, would be much reduced or increased, respectively. For example, mallards are known to pair up during autumn or winter (depending on latitude; Bezzel, 1959; Raitasuo, 1964). However, we did not find a signal of this behaviour in our data, even though this period is characterized by courtship behaviours that potentially could have increased the mean activity we measured. Possibly, pairing up is spread over too long a period (Raitasuo, 1964) so that the average activity over time does not show a peak.

The only measured weather factor that showed a clear and consistent effect on activity was rain: activity strongly increased with rain. However, we are unable to determine what type of activity correlated with rain. One hypothesis is that the ducks were mainly passive when it was raining, but regularly shook off rain drops, yielding increased acceleration measurements in the tag. On the other hand, rain may have induced feeding, for example because of softening of the ground, wetting of grains on the fields or availability of puddles; Pernollet et al. (2015) found that dabbling duck species profit from flooded (compared to dry) rice fields, due to better feeding opportunities. Mallards appear to drink regularly while feeding, especially when food is soiled with nondigestible matter (Guillemain, Corbin, \& Fritz, 1999; Thomas, 1981). We have another indication of the mallards being truly more active during rain: the raw average flight probability per rain class slightly increased during soft or moderate rain compared to no rain, from around $1.0 \%$ without rain to around $1.3 \%$ during $0.75 \mathrm{~mm} / \mathrm{h}$ rain (Table A2). Waddling showed a similar pattern, increasing from about $2 \%-3 \%$ for the same rain classes (waddling was tentatively identified for the data from Lake Sempach, only; Fig. A3). Paulus (1988), on the other hand, reported that feeding tended to decrease with rain (and high wind speed) in three dabbling duck species (no data on mallards given).

Temperature and wind speed, in contrast, showed less consistency in their effect on mallard activity in our data. Guillemain et al. (2002) observed increased feeding activity of different granivorous duck species (the mallard among them) in colder weather: the usual night feeding was supplemented with daytime feeding when it was cold. We only found a weak signal of temperature on activity in our data, but it was consistent with Guillemain et al. (2002): more activity during the day at colder 


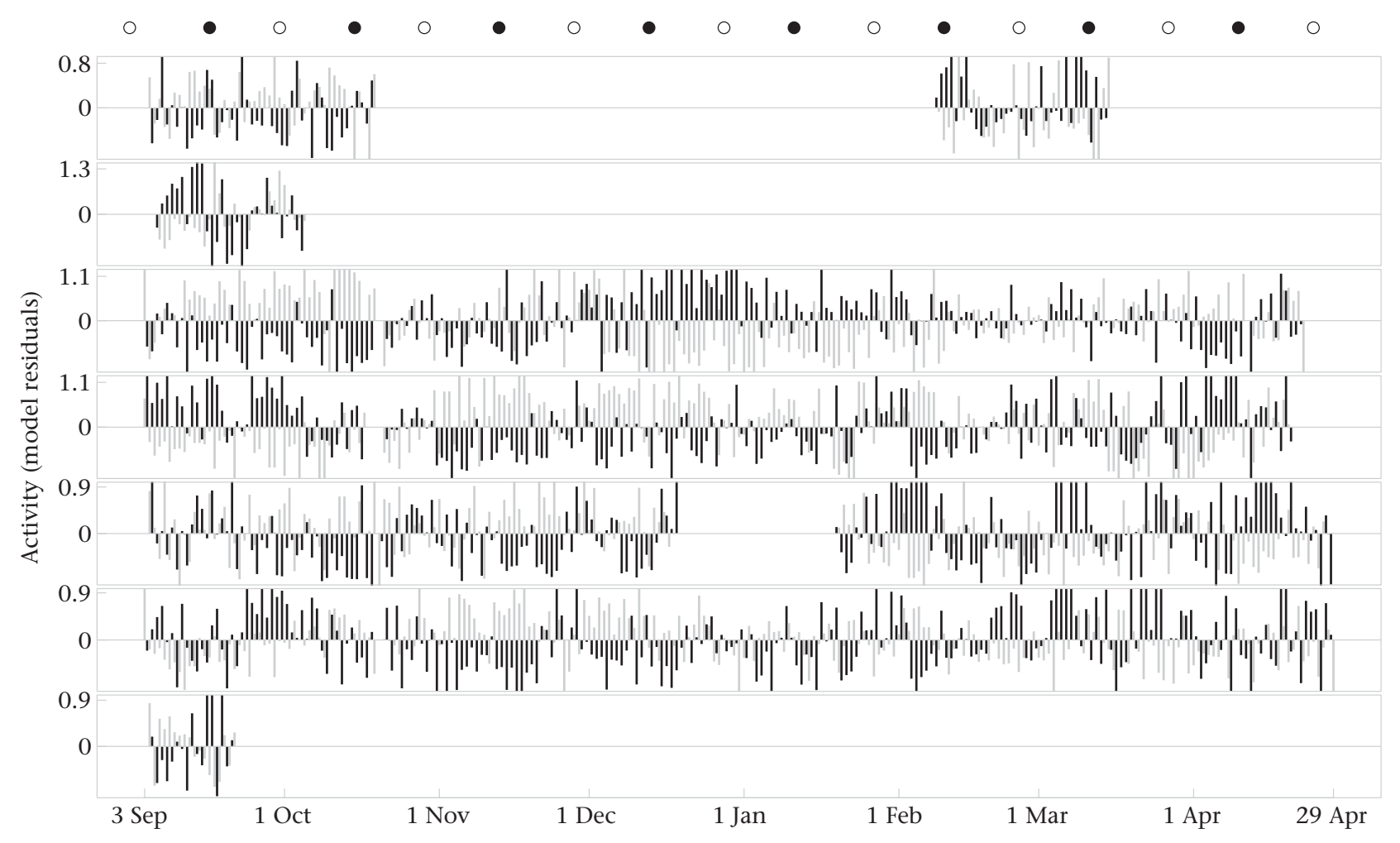

Figure 6. As Fig. 5, but for the ducks from Lake Constance (one individual followed only during January 2012 is not depicted).

temperatures (found in the Lake Constance data). However, our data do not support a clear and consistent quadratic temperature effect (increasing feeding activity with lower nonfreezing temperatures, then decreasing activity with freezing temperatures; Paulus, 1988), especially not a clear reduction of activity below $0{ }^{\circ} \mathrm{C}$. Hence, our data may better match with Bossenmair and Marshall (1958) who reported that feeding flights of waterbirds were observed during any weather situation, even during dense fog and blizzards. Note that we measured the overall activity level, while most studies on activity changes with temperature focused on a specific activity (often feeding).

The ducks from Lake Sempach appeared to be more active during a full moon as well as during a new moon, and this pattern was independent of whether the moon was above or below the horizon. While increased activity during moonlight nights has been described repeatedly (McNeil et al., 1992), increased activity during new moon nights has not been reported in ducks, to our knowledge. One tentative explanation for this observation could be increased alert during darkness due to predators (e.g. red fox Vulpes vulpes). Increased insect activity during a new moon has been described (e.g. Williams, Singh, \& El Ziady, 1956), and waterbirds may feed on insects (Ydenberg et al., 1984), which could lead to increased duck activity during a new moon. However, insects on land were probably inactive during our winter study period at Lake Sempach, although ducks may have foraged on aquatic invertebrates (Guillemain, Fritz, \& Guillon, 2000). In the data from Lake Constance the moon effects were much less pronounced, with a small increase in activity during times with more moonlight, the expected result, but no indication of more activity during a new moon. Of course, the situation in the Lake Constance study area may have been different, such that an increased activity during the darkest night periods, possibly due to increased vigilance, remains a hypothesis to be considered in future work.
The analyses based on the residuals per half-day revealed two aspects. First, an increase in activity during daylight correlated with a decrease in activity during the night and vice versa, yielding a conspicuous $12 \mathrm{~h}$ alternating pattern in the residuals of activity for the half-day, and a mostly irregular switching between excess daytime activity to excess night activity over a few days. Second, we observed a lack of synchrony of this pattern among individuals. For the time being, we can only speculate about the reasons for this finding. Thereby, we need to keep in mind that each duck was probably a member of a group of ducks; hence the timing of activity and rest was probably codetermined by group decision mechanisms, in addition to the physiological state and motivation of the individual.

In the Lake Sempach data, the switching between excess daytime activity and excess night activity had a frequency of approximately 7 days in some individuals. This rhythm matches the moon cycle and with the observed correlation of increased activity twice per moon cycle (during new and full moon, see above). But the pattern was not universal, as other individuals did not show such a 7-day rhythm. Excess activity during either half-day may be linked to some food source and the distribution of disturbances. For example, if mallards prefer to feed (or otherwise to be active) during the day, they can do that as long as they stay and feed in undisturbed areas. But when they change to feed, for example on fields where disturbance during the day may be common (hikers, dogs, etc.), the ducks might switch to excess night activity. It has been shown that mallards, after discovering a food source such as a field, often use this food source for a number of consecutive days before switching to a new food source (Bossenmair \& Marshall, 1958; Thomas, 1981). Possibly, this behaviour is mirrored in our activity data.

The irregular pattern of excess daylight or night activity and the lack of apparent synchrony among individuals suggest that different variables from those that we measured (three weather parameters, moon parameters, season) modulate the basic, rather constant diel 
activity pattern of the mallard. In a flexible species such as the mallard these variables probably interact in a complex way and vary in space and time, e.g. availability of food, presence of disturbances, behaviour of other mallards in the area, etc. A similar independence from weather and moon conditions was described for the common raven, Corvus corax, another extremely flexible species (Janicke \& Chakarov, 2007). On the other hand, the switching between excess daylight and night activity every few days could also be (partially) independent of external factors; instead it could be an effect of an innate clock that is not in phase with the day-night rhythm. Raitasuo (1964) stated that mallards (as other Anas species) typically show phases of increased and decreased activity alternating about every $30-75 \mathrm{~min}$. This could, for example, be related to a feeding-digesting rhythm, i.e. a physiological driver. Daan and Aschoff (1975) and Kronfeld-Schor and Dayan (2003) both discussed cases of multiple, nonsynchronous oscillators in various animals. Our data may manifest another such example, with a fixed diel activity pattern entrained by the sun, overlain by another oscillator (with unknown drivers), resulting in the regular switching between excess day and night activity.

\section{Acknowledgments}

We thank Josef Hofer, Stefan Felder, Emmanuel Rey and Daniel Wili for help in the field. David Rodrigues, Andreas Schmidt, Doris Matthes, Martin Grüebler, Beat Naef-Danzer, Vreni Mattmann and Dieter Peter provided help with the tags and the tagging. We received GIS support from Jérôme Guélat and Gabriele Hilke and valuable feedback on the study from Verena Keller. The study, originally designed to investigate short-distance movements of mallards in winter, was financially supported by the Swiss Federal Food Safety and Veterinary Office FSVO. Project number 1.09.02.

\section{References}

Arzel, C., Elmberg, J., \& Guillemain, M. (2007). A flyway perspective of foraging activity in Eurasian Green-winged Teal, Anas crecca crecca. Canadian Journal of Zoology, 85, 81-91. http://dx.doi.org/10.1139/Z06-201.

Bauer, H. G. Bezzel, E \& Fiedler, W (2005). Anas platyrhynchos-Stockente. In H. G. Bauer, E. Bezzel, \& W. Fiedler (Eds.), Kompendium der Vögel Mitteleuropas (pp. 93-97). Wiebelsheim, Germany: Aula.

Bengtsson, D., Avril, A., Gunnarsson, G., Elmberg, J., Söderquist, P., Norevik, G., et al. (2014). Movements, home-range size and habitat selection of Mallards during autumn migration. Plos One, 9, e100764. http://dx.doi.org/10.1371/ journal.pone.0100764.

Bezzel, E. (1959). Beiträge zur Biologie der Geschlechter bei Entenvögeln. Anzeiger der ornithologischen Gesellschaft in Bayern, 5, 269-355.

Bossenmair, E. F., \& Marshall, W. H. (1958). Field-feeding by waterfowl in southwestern Manitoba. Wildlife Monographs, 1, 1-32.

Daan, S., \& Aschoff, J. (1975). Circadian rhythms of locomotor activity in captive birds and mammals: their variations with season and latitude. Oecologia, 18, 269-316.

Diefenbach, D. R., Nichols, J. D., \& Hines, J. E. (1988). Distribution patterns of American black duck and mallard winter band recoveries. The Journal of Wildlife Management, 52, 704-710. http://dx.doi.org/10.2307/3800934.

Dunlap, J. C., Loros, J. J., \& DeCoursey, P. J. (Eds.). (2004). Chronobiology: Biological timekeeping. Sunderland, MA: Sinauer Associates.

Guillemain, M., Corbin, J., \& Fritz, H. (1999). Interruptions of terrestrial feeding as a way to decrease the non-digestible fraction of the bolus: field observations and laboratory experiments in Mallard. Wildfowl, 50, 123-132.

Guillemain, M., Fritz, H., \& Duncan, P. (2002). Foraging strategies of granivorous dabbling ducks wintering in protected areas of the French Atlantic coast. Biodiversity and Conservation, 11, 1721-1732. http://dx.doi.org/10.1023/A: 1020322032114.

Guillemain, M., Fritz, H., \& Guillon, N. (2000). Foraging behavior and habitat choice of wintering northern shoveler in a major wintering quarter in France. Waterbirds, 23, 355-363. http://dx.doi.org/10.2307/1522173.

Gwinner, E. (1975). Circadian and circannual rhythms in birds. In D. S. Farner, \& J. R. King (Eds.), Avian biology (Vol. 5), pp. 221-285). New York, NY: Academic Press.

Hofer, J., Korner, P., \& Korner-Nievergelt, F. (2010). Auftreten und Herkunft der Wasservögel am Sempachersee. Ornithologischer Beobachter, 11 (Supplementary issue).
Janicke, T., \& Chakarov, N. (2007). Effect of weather conditions on the communal roosting behaviour of common ravens Corvus corax with unlimited food resources. Journal of Ethology, 25, 71-78. http://dx.doi.org/10.1007/s10164-0060209-3.

Johnson, W. P., Schmidt, P. M., \& Tylor, D. P. (2014). Foraging flight distances of wintering ducks and geese: a review. Avian Conservation and Ecology, 9(2), 2. http://dx.doi.org/10.5751/ACE-00683-090202.

Korner, P., Sauter, A., \& Jenni, L. (2015). Raum-zeitliches Verhalten von neun Stockenten Anas platyrhynchos im Winter. Ornithologischer Beobachter, 112, 77-96.

Kozulin, A. (1995). Ecology of Mallards Anas platyrhynchos wintering in low temperatures conditions in Belarus. Acta Ornithologica, 30, 125-134.

Kronfeld-Schor, N., \& Dayan, T. (2003). Partitioning of time as an ecological resource. Annual Review of Ecology, Evolution, and Systematics, 34 $153-181$.

Martin, G. (1990). Birds by night. London, U.K.: T \& AD Poyser.

Mayhew, P. W. (1988). The daily energy intake of European Wigeon in winter. Ornis Scandinavica, 19, 217-223. http://dx.doi.org/10.2307/3676562.

McNeil, R., Drapeau, P., \& Goss-Custard, J. D. (1992). The occurrence and adaptive significance of nocturnal habits in waterfowl. Biological Reviews, 67, 381-419. http://dx.doi.org/10.1111/j.1469-185X.1992.tb01188.x.

Merke, F. R., \& Mosbech, A. (2008). Diurnal and nocturnal feeding strategies in Common Eiders. Waterbirds, 31, 580-586. http://dx.doi.org/10.1675/1524-469531.4.580.

Owen, M. (1991). Nocturnal feeding in waterfowl. In B. D. Bell (Ed.), Acta XX Congressus Internationalis Ornithologici (pp. 1105-1112). Christchurch, New Zealand: New Zealand Ornithological Congress Trust Board.

Paulus, S. L. (1988). Time-activity budgets of nonbreeding Anatidae: a review. In L. Wennerberg (Ed.), Waterfowl in Winter (pp. 135-152). Minneapolis, MN University of Minnesota.

Pernollet C. A., Guelmami, A. Green, A. J., Curco Masip, A., Dies, B., Bogliani, G., et al. (2015). A comparison of wintering duck numbers among European rice production areas with contrasting flooding regimes. Biological Conservation, 186 214-224. http://dx.doi.org/10.1016/j.biocon.2015.03.019.

Pulliainen, E. (1963). On the history, ecology and ethology of the Mallards (Anas platyrhynchos L) overwintering in Finland. Ornis Fennica, 2, 45-65.

Quinlan, E. E., \& Baldassarre, G. A. (1984). Activity budgets of nonbreeding Green winged Teal on Playa Lakes in Texas. The Journal of Wildlife Management, 48 838-845. http://dx.doi.org/10.2307/3801430.

R Core Team. (2015). R: A language and environment for statistical computing. Vienna, Austria: R Foundation for Statistical Computing. www.R-project.org.

Raitasuo, K. (1964). Social behaviour of the mallard, Anas platyrhynchos, in the course of the annual cycle. Papers on Game Research, 24, 1-72.

Roshier, D. A., Klomp, N. I., \& Asmus, M. (2006). Movements of a nomadic waterfowl, Grey Teal, Anas garcilis, across inland Australia-results from satellite telemetry spanning fifteen months. Ardea, 94, 460-475.

Sauter, A., Korner-Nievergelt, F., \& Jenni, L. (2010). Evidence of climate change effects on within-winter movements of European Mallards Anas platyrhynchos. Ibis, 152, 600-609. http://dx.doi.org/10.1111/j.1474-919X.2010.01028.x.

Sauter, A., Korner P., Fiedler, W. \& Jenni, L. (2012). Individual behavioural variability of an ecological generalist: activity patterns and local movements of Mallards Anas platyrhynchos in winter. Journal of Ornithology, 153, 713-726. http:// dx.doi.org/10.1007/s10336-011-0788-9.

Scott, D. A., \& Rose, P. M. (1996). Atlas of Anatidae Populations in Africa and Western Eurasia. Oxford, U.K.: Wetlands International.

Sharma, V. K., \& Chandrashekaran, M. K. (2005). Zeitgebers (time cues) for biological clocks. Current Science, 89, 1136-1146.

Shepard, E. L. C., Wilson, R. P., Quintana, F., Gómez Laich, A., Liebsch, N., Albareda, D. A., et al. (2008). Identification of animal movement patterns using tri-axial accelerometry. Endangered Species Research, 10, 47-60.

Tamisier, A. (1978). The functional units of wintering ducks: a spatial integration of their comfort and feeding requirements. Verhandlungen der ornithologischen Gesellschaft in Bayern, 23, 229-238.

Thomas, G. J. (1981). Field feeding by dabbling ducks around the Ouse Washes, England. Wildfowl, 32, 69-78.

Thomas, G. J. (1982). Autumn and winter feeding ecology of waterfowl at the Ouse Washes England. Journal of Zoology, 197, 131-172.

van Toor, M. L., Hedenström, A., Waldenström, J., Fiedler, W., Holland, R. A Thorup, K., et al (2013). Flexibility of continental navigation and migration in European Mallards. PLOS ONE, 8, e72629. http://dx.doi.org/10.1371/ journal.pone.0072629.

Williams, C. B., Singh, B. P., \& El Ziady, S. (1956). An investigation into the possible effects of moonlight on the activity of insects in the field. Proceedings of the Royal Entomological Society of London. Series A, 31, 135-144.

Winner, R. W. (1960). Fall and winter movements of black and Mallard ducks. The Journal of Wildlife Management, 24, 332-335.

Ydenberg, R. C., Prins, H. H. T., \& Van Dijk, J. (1984). A lunar rhythm in the nocturnal foraging activities of wintering Barnacle Geese. Wildfowl, 35, 93-96.

Zavalaga, C. B., Dell'Omo, G., Becciu, P., \& Yoda, K. (2011). Patterns of GPS tracks suggest nocturnal foraging by incubating Peruvian Pelicans (Pelecanus thagus). Plos One, 6, e19966. http://dx.doi.org/10.1371/journal.pone.0019966. 


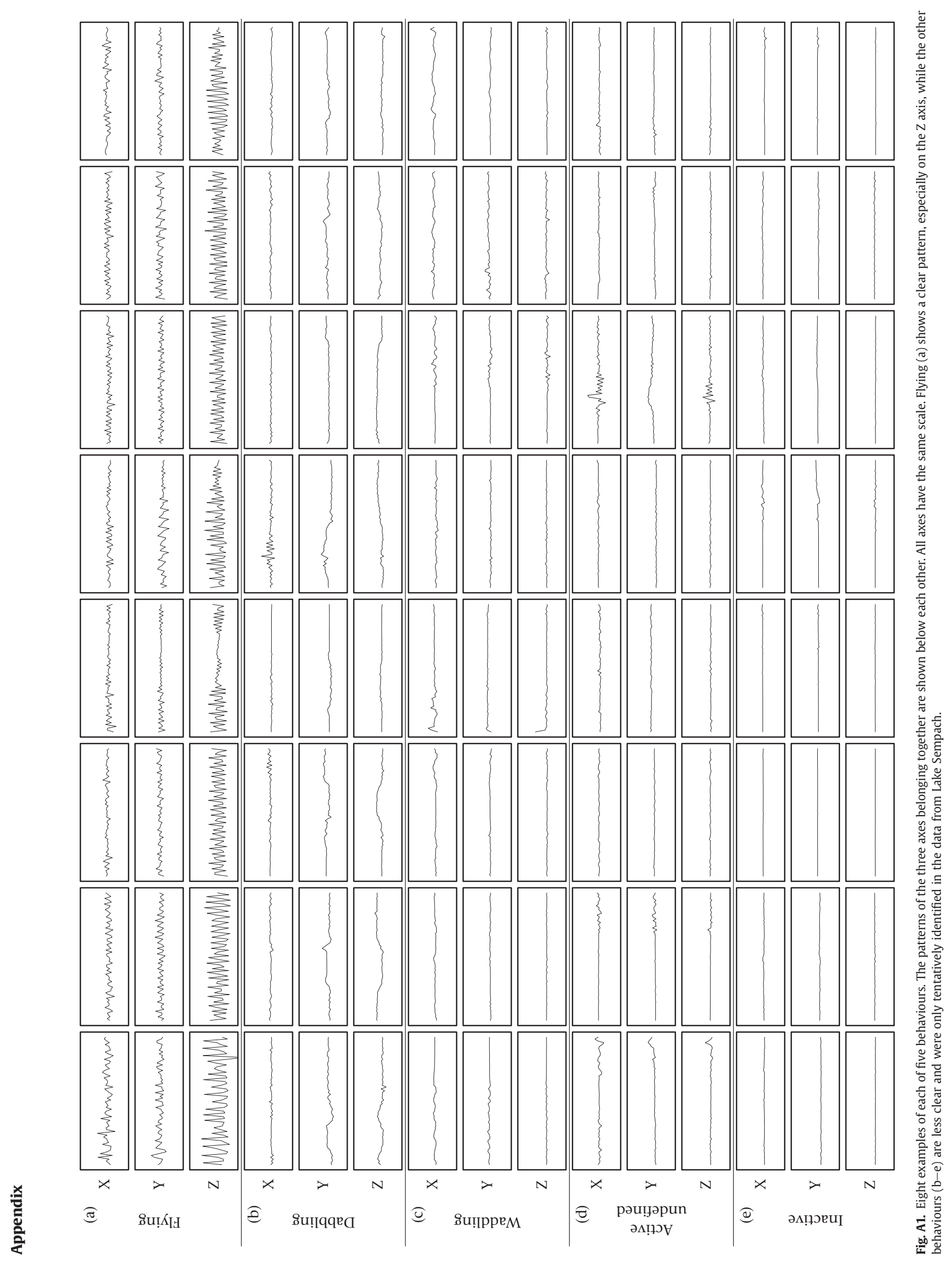




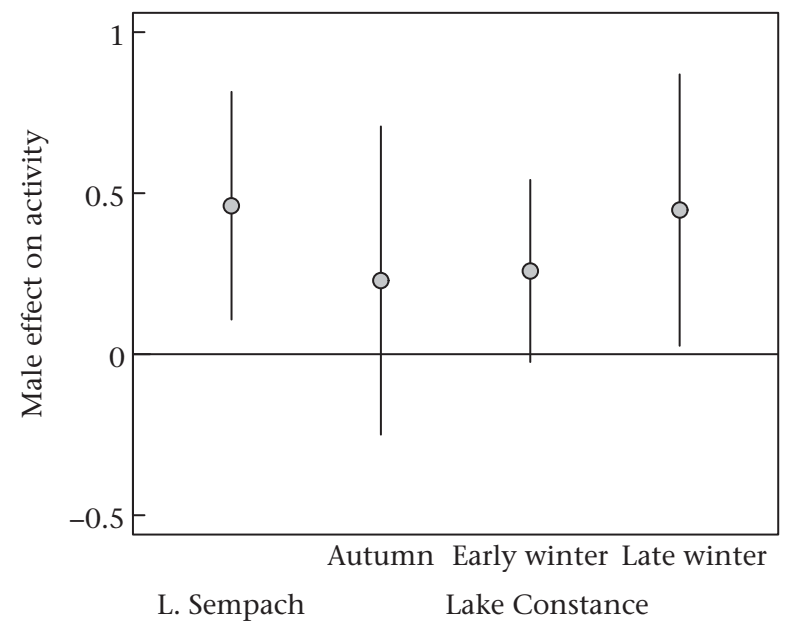

Fig. A2. Effect of sex on the overall activity level. Separate models were fitted for the data from Lake Sempach and for three periods from Lake Constance. The model estimated the mean activity per day period and per sex, while individual and date were random factors; the model included a temporal autocorrelation correction on the residuals. Vertical bars are approximate $95 \%$ confidence intervals.

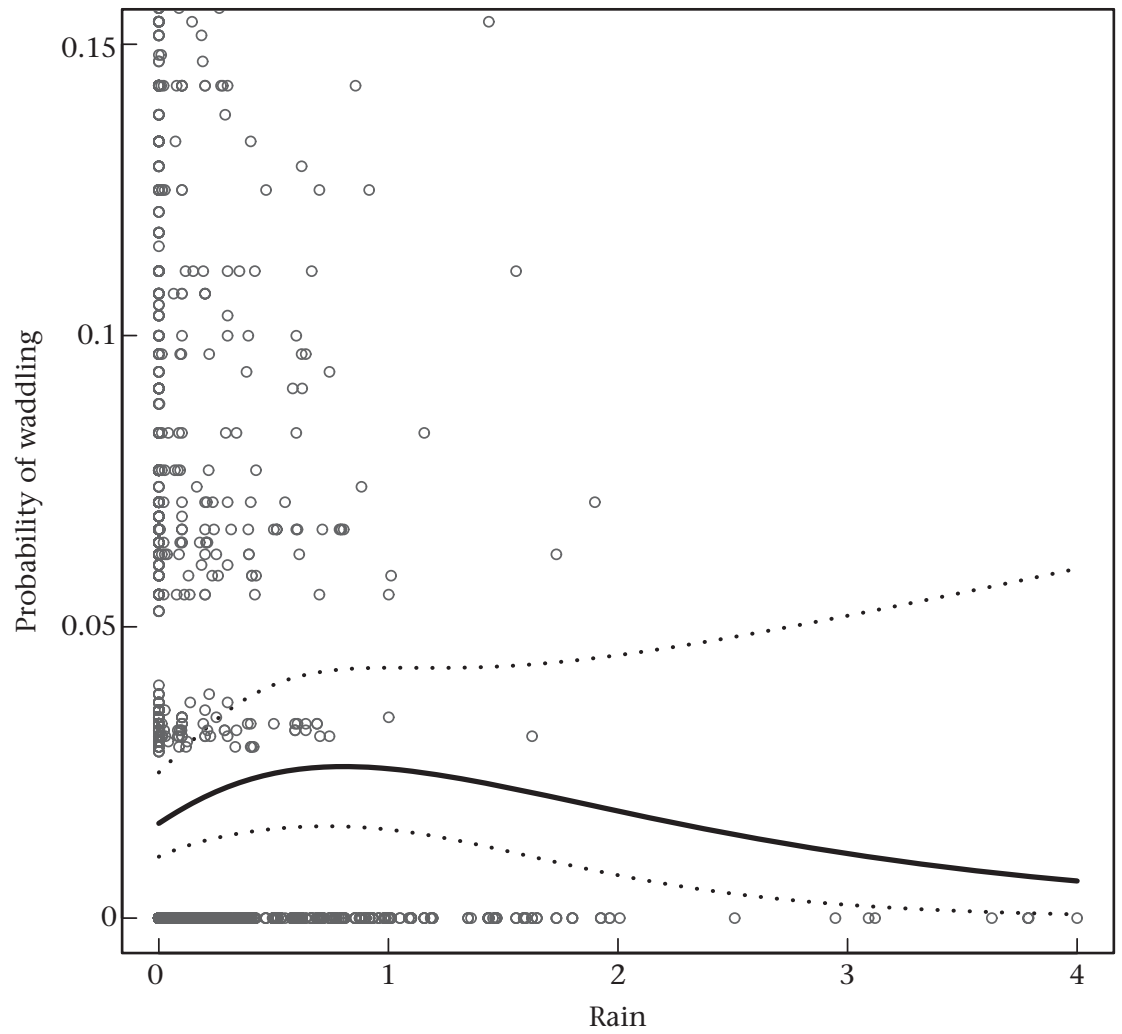

Fig. A3. Relationship between waddling and rain ( $\mathrm{mm} / \mathrm{h}$ ). Waddling was tentatively identified in the data from Lake Sempach, only. The solid line is the estimated probability from a binomial model (correcting for autocorrelation, with random factors individual and day period; rain and rain ${ }^{2}$, wind and temperature as fixed predictors; dotted lines indicate an approximate $95 \%$ confidence interval). Small circles are the observed waddling proportions per day period (there were 225 points with waddling proportions were between 0.15 and about 0.4 and, therefore, are not visible in the plot; 199 of these observations were at rain $<0.5$ ). 
Table A1

Moon and weather effects on the overall mallard activity, estimated separately for the two study sites and for the two half-days night and daylight

\begin{tabular}{|c|c|c|c|c|}
\hline Predictors & Transformation, levels and/or unit & Estimate (SE) & $P$ & Selected fitted values [95\% confidence interval] \\
\hline \multicolumn{5}{|l|}{ Model I } \\
\hline Intercept & - & $4.700(0.356)$ & $<0.001$ & - \\
\hline Temperature & $z$-transformed, hourly average, ${ }^{\circ} \mathrm{C}$ & $0.085(0.064)$ & 0.183 & $\begin{array}{l}-5^{\circ} \mathrm{C}: 4.62[3.96 ; 5.28] \\
+5^{\circ} \mathrm{C}: 4.86[4.15 ; 5.57]\end{array}$ \\
\hline Rain & Log (rain +1 ), hourly sum, mm & $1.046(0.269)$ & $<0.001$ & $\begin{array}{l}0 \mathrm{~mm}: 4.69[4.05 ; 5.33] \\
2 \mathrm{~mm}: 5.84[4.98 ; 6.70] \\
\text { (compare also Fig. } 3 \text { ) }\end{array}$ \\
\hline Wind & Log (wind), hourly mean, km/s & $-0.039(0.128)$ & 0.762 & Very small effect \\
\hline Moon sickle & $-0.5=$ new moon, $0=$ half - moon, $+0.5=$ full moon & $-0.087(0.196)$ & 0.657 & $\begin{array}{l}\text { Effect depicted in Fig. } 4 \text { New moon: } 5.37[4.71 ; 6.02] \\
\text { Half-moon: } 4.77 \text { [4.13; } 5.41] \text { Full moon: } 5.28[4.57 ; 5.99\end{array}$ \\
\hline Moon sickle squared & - & $2.226(0.706)$ & 0.002 & \\
\hline \multirow[t]{2}{*}{ Moon position } & Below horizon (baseline) & - & - & - \\
\hline & Above horizon & $0.182(0.094)$ & 0.053 & Effect depicted in Fig. 4 \\
\hline \multicolumn{5}{|l|}{ Model II } \\
\hline Intercept & - & $6.244(0.282)$ & $<0.001$ & - \\
\hline Temperature & $z$-transformed, hourly average, ${ }^{\circ} \mathrm{C}$ & $-0.015(0.054)$ & 0.787 & Very small effect \\
\hline Rain & $\log ($ rain +1$)$, hourly sum, mm & $0.762(0.231)$ & 0.001 & $\begin{array}{l}0 \mathrm{~mm}: 6.20[5.69 ; 6.71] \\
2 \mathrm{~mm}: 7.04[6.34 ; 7.74] \\
\text { (compare also Fig. } 3 \text { ) }\end{array}$ \\
\hline Wind & Log (wind), hourly mean, $\mathrm{km} / \mathrm{s}$ & $-0.144(0.107)$ & 0.179 & Very small effect \\
\hline \multicolumn{5}{|l|}{ Model III } \\
\hline Intercept & - & $4.566(0.175)$ & $<0.001$ & - \\
\hline Temperature & $z$-transformed, hourly average, ${ }^{\circ} \mathrm{C}$ & $-0.039(0.029)$ & 0.187 & Small effect \\
\hline Rain & $\log ($ rain +1$)$, hourly sum, $\mathrm{mm}$ & $0.980(0.082)$ & $<0.001$ & $\begin{array}{l}\text { Effect depicted in Fig. } 3 \\
0 \mathrm{~mm}: 4.76[4.46 ; 5.05] \\
2 \mathrm{~mm}: 5.84[5.50 ; 6.18]\end{array}$ \\
\hline Wind & Log (wind), hourly mean, km/s & $0.079(0.040)$ & 0.046 & Very small effect \\
\hline Moon sickle & $-0.5=$ new moon, $0=$ half-moon, $+0.5=$ full moon & $-0.123(0.117)$ & 0.292 & Effect depicted in Fig. 4 \\
\hline \multirow[t]{2}{*}{ Moon position } & Below horizon (baseline) & - & - & New moon below horizon: 4.94 [4.64; 5.24] \\
\hline & Above horizon & $0.159(0.041)$ & $<0.001$ & New moon above horizon: $4.88[4.55 ; 5.20]$ \\
\hline \multirow[t]{2}{*}{ Moon sickle $\mathrm{x}$ moon position } & Sickle $\times$ below horizon (baseline) & - & - & Half-moon below horizon: $4.88[4.58 ; 5.18]$ \\
\hline & Sickle $\times$ above horizon & $0.454(0.145)$ & 0.002 & $\begin{array}{l}\text { Half-moon above horizon: } 5.04[4.74 ; 5.33] \\
\text { Full moon below horizon: } 4.82[4.48 ; 5.16] \\
\text { Full moon above horizon: } 5.21[4.90 ; 5.51]\end{array}$ \\
\hline \multicolumn{5}{|l|}{ Model IV } \\
\hline Intercept & - & $6.039(0.240)$ & $<0.001$ & - \\
\hline Temperature & $z$-transformed, hourly average, ${ }^{\circ} \mathrm{C}$ & $-0.054(0.025)$ & 0.028 & $-10^{\circ} \mathrm{C}: 6.03[5.51 ; 6.56]$ \\
\hline Temperature squared & - & $-0.047(0.017)$ & 0.007 & $\begin{array}{l}5^{\circ} \mathrm{C}: 6.22[5.77 ; 6.67] \\
20^{\circ} \mathrm{C}: 5.90[5.44 ; 6.37]\end{array}$ \\
\hline Rain & Log (rain +1 ), hourly sum, mm & $0.793(0.093)$ & $<0.001$ & $\begin{array}{l}0 \mathrm{~mm}: 6.14[5.69 ; 6.59] \\
2 \mathrm{~mm}: 7.02[6.53 ; 7.51] \\
\text { (compare also Fig. } 3 \text { ) }\end{array}$ \\
\hline Wind & Log (wind), hourly mean, $\mathrm{km} / \mathrm{s}$ & $0.044(0.035)$ & 0.214 & Very small effect \\
\hline
\end{tabular}

Model I: Lake Sempach during the night. Estimated autocorrelation $\Phi=0.355$. Sample size $=2142$ cases, 7 individuals, 69 dates, 10 time levels. Model II: Lake Sempach during daylight. Estimated autocorrelation $\Phi=0.241$. Sample size $=2123$ cases, 7 individuals, 70 dates, 10 time levels. Model III: Lake Constance during the night. Estimated autocorrelation $\Phi=0.353$. Sample size $=9198$ cases, 8 individuals, 206 dates, 10 time levels. Model IV: Lake Constance during daylight. Estimated autocorrelation $\Phi=0.261$. Sample size = 10259 cases, 8 individuals, 239 dates, 10 time levels. The outcome variable is the mean activity per day period (10ths of the night or daylight, respectively); most activity values were between 3 and 8 (see Figs. 3 and 4 for corresponding histograms). Selected fitted values are given in the last column to illustrate the effects (the other covariables are set to the average value and factors to the baseline level); the uncertainties of the fitted values are calculated applying the delta method. Starting models also included squared effects of temperature and moon sickle, as well as the interaction between moon sickle and moon position, but these effects were dropped when nonsignificant. In all models, duck individual, date and day period were included as random factors.

Table A2

Percentage of bouts classified as flights, depending on rain classes and separately for the two study sites and seasons

\begin{tabular}{|c|c|c|c|c|c|c|c|}
\hline \multirow[t]{2}{*}{ Site } & \multirow[t]{2}{*}{ Season } & \multicolumn{6}{|c|}{ Rain classes $(\mathrm{mm} / \mathrm{h})$} \\
\hline & & $0-0.25$ & $0.25-0.5$ & $0.5-0.75$ & $0.75-1$ & $1-1.25$ & $1.25-1.5$ \\
\hline Lake Sempach & 19 January - 29 March & 1.1 & 1.7 & 1.3 & 1.8 & 0.7 & 0 \\
\hline Lake Constance & 4 September - 11 November & 0.9 & 1.0 & 0.8 & 0.6 & 0.3 & 0.3 \\
\hline Lake Constance & 12 November - 18 January & 0.7 & 0.8 & 1.7 & 1.0 & 0.3 & 0 \\
\hline Lake Constance & 19 January - 29 March & 1.4 & 0.5 & 0.9 & 2.1 & 1.0 & 0.9 \\
\hline Arithmetic mean & & 1.0 & 1.0 & 1.2 & 1.4 & 0.6 & 0.3 \\
\hline
\end{tabular}

Rain $>1.5 \mathrm{~mm} / \mathrm{h}$ was observed in only $1-2 \%$ of the day periods; hence there are not enough data to describe the duck flying behaviour during heavy rain. 